\title{
Stochastic Theory of Ultrathin Lubricant Film Melting in the Stick-Slip Regime
}

\author{
A. V. Khomenko and I. A. Lyashenko \\ Sumy State University, Sumy, 40007 Ukraine \\ e-mail:khom@phe.sumdu.edu.ua \\ Received October 4, 2004
}

\begin{abstract}
Melting of an ultrathin lubricant film under friction between atomically smooth surfaces is studied in terms of the Lorentz model. Additive noise associated with shear stresses and strains, as well as with film temperature, is introduced, and a phase diagram is constructed where the noise intensity of the film temperature and the temperature of rubbing surfaces define the domains of sliding, dry, and stick-slip friction. Conditions are found under which stick-slip friction proceeds in the intermittent regime, which is characteristic of selforganized criticality. The stress self-similar distribution, which is provided by temperature fluctuations, is represented with allowance for nonlinear relaxation of stresses and fractional feedbacks in the Lorentz system. Such a fractional scheme is used to construct a phase diagram separating out different types of friction. Based on the study of the fractional Fokker-Planck equation, the conclusion is drawn that stick-slip friction corresponds to the subdiffusion process. (C) 2005 Pleiades Publishing, Inc.
\end{abstract}

\section{INTRODUCTION}

Sliding friction of smooth solid surfaces with a thin lubricant film in between has recently become a subject of increased interest [1]. One reason is that weakly rubbing surfaces are widely applied in a variety of advanced high-tech products, such as computer memories, miniature engines, and space-borne devices. A great insight into the physics of friction has been provided by experiments with atomically smooth mica surfaces separated by an ultrathin organic lubricant layer, which behaves as a solid under certain conditions [2]. Specifically, intermittent (stick-slip) motion was observed [3-6], which is inherent in dry friction and causes wear and failure of rubbing parts. Such "mixed" friction conditions arise when a lubricant film less than four molecular layers thick solidifies, being compressed by the walls. The subsequent abrupt transition to melting takes place when the shear stress exceeds a critical value (melting due to shear).

Thus, thin molecular films experience the transition from the solid-like to liquid-like phase $[2,7]$, the properties of the latter being impossible to describe even qualitatively in the terms (e.g., viscosity) characterizing the properties of a normal liquid occupying a large volume. Such films exhibit a yield stress, which is a characteristic of failure in solids, while the times of molecular diffusion and relaxation in them may by more than 10 orders exceed the corresponding values for a normal liquid or even films that are a little bit thicker.

Investigation of the effect of noise (fluctuations) on sliding friction is also of great fundamental and applied significance, since in real experiments, fluctuations critically affect the frictional behavior, for example, reduce the friction $[1,8,9]$. Thermal noise, observed in any experiments, may carry an ultrathin lubricant film from the stable solid-like state to the liquid-like state and thus, transform dry friction into sliding or stick-slip friction. Therefore, considerable attention has recently been given to the effect of noise and uncontrolled impurities present at the friction boundary on static and dynamic friction [10-12]. It has been shown that surfaces with a regular (periodical) relief have a lower friction coefficient than irregular surfaces.

Earlier [13, 14], we elaborated upon an idea that the solid-liquid transition of an ultrathin lubricant film is the result of thermodynamic and shear melting. The associated processes were considered in terms of selforganization of shear stress and strain fields, as well as of the lubricant film temperature, with allowance for the additive noise of these quantities (Sect. 1). However, the issue as to whether self-organized criticality (SOC) [15] may occur in the system was left aside. In this work, we try to find conditions for SOC using the Lorentz model, which gives a field representation of a elastoviscous medium [16].

The SOC conditions arise in the case of a powertype stress distribution with a fractional exponent. Therefore, in Sect. 2, we generalize (modify) the Lorentz system in order to describe, in accordance with experimental data [2], intermittent melting of a lubricant film and, consequently, the related friction conditions. By analogy with [14], we construct a phase diagram that allows one to trace changes in the domains of sliding, dry, and stick-slip friction depending on the fractional exponent in the modified Lorentz system. With such a generalization, we describe the behavior of the system in terms of nonadditive thermodynamics 
[17] (Sect. 3). Such an approach can be implemented in the fractional Lorentz system, where the stress serves as the order parameter, the conjugate field is reduced to nonadditive complexity, and the internal energy is a control parameter. Eventually, it turns out that the stress distribution inherent in SOC is provided by energy fluctuations. This distribution is, on the one hand, a solution to the nonlinear Fokker-Planck equation describing the behavior of a nonadditive system [17] and, on the other hand, stems from the fractional Fokker-Planck equation for Levi flights [18]. Contrasting solutions to these equations, one can establish correlations between the exponent in the stress distribution (the characteristic exponent of multiplicative noise), fractal dimension of the phase space, number of equations needed to describe the self-consistent behavior of the system under the SOC conditions, dynamic exponent, and Tsallis nonadditivity parameter. It is shown that stickslip friction corresponds to the subdiffusion process.

\section{BASIC EQUATIONS AND THE EFFECT OF NOISE}

Rheologically describing a viscoelastic heat-conducting medium [13], we derived a set of kinetic equations governing the consistent behavior of shear stresses $\sigma$, strains $\varepsilon$, and temperature $T$ in an ultrathin lubricant film between atomically smooth rubbing mica surfaces. Let us write these equations for $\sigma, \varepsilon$, and $T$ using the following units of measure:

$$
\begin{gathered}
\sigma_{s}=\left(\frac{\rho c_{\mathrm{v}} \eta_{0} T_{\mathrm{c}}}{\tau_{\mathrm{h}}}\right)^{1 / 2}, \\
\varepsilon_{s}=\frac{\sigma_{s}}{G_{0}} \equiv\left(\frac{\tau_{\varepsilon}}{\tau_{\mathrm{h}}}\right)^{1 / 2}\left(\frac{\rho c_{\mathrm{v}} T_{\mathrm{c}} \tau_{\varepsilon}}{\eta_{0}}\right)^{1 / 2},
\end{gathered}
$$

where $\rho$ is the lubricant density, $c_{V}$ is the specific heat at constant volume, $T_{\mathrm{c}}$ is the critical temperature, $\eta_{0} \equiv$ $\eta\left(T=2 T_{\mathrm{c}}\right)$ is the characteristic value of shear viscosity $\eta, \tau_{\mathrm{h}} \equiv \rho l^{2} c_{v} / \kappa$ is the heat conduction time, $l$ is the heat conduction length, $\kappa$ is the thermal conductivity, $\tau_{\varepsilon}$ is the strain relaxation time, and $G_{0} \equiv \eta_{0} / \tau_{\varepsilon}$. The corresponding equations are

$$
\begin{gathered}
\tau_{\sigma} \dot{\sigma}=-\sigma+g \varepsilon, \\
\tau_{\varepsilon} \dot{\varepsilon}=-\varepsilon+(T-1) \sigma, \\
\tau_{\mathrm{h}} \dot{T}=\left(T_{\mathrm{m}}-T\right)-\sigma \varepsilon+\sigma^{2} .
\end{gathered}
$$

Here, $\tau_{\sigma}$ is the stress relaxation time, $T_{\mathrm{m}}$ is the temperature of atomically smooth rubbing mica surfaces, $g=$ $G / G_{0}<1$ is a constant, and $G$ is the shear modulus of the lubricant. Equation (2) is reduced to the Maxwell equation for a viscoelastic medium by substituting $\varepsilon / \tau_{\sigma}$ for $\partial \varepsilon / \partial t$. Expression (3) is similar to the Kelvin-Voigt equation [13, 19], which takes into account the dependence of the shear viscosity on dimensionless tempera- ture $\eta=\eta_{0} /(T-1)$. Expression (4) is the heat conduction expression that involves heat transfer from rubbing surfaces to a lubricant film, dissipative heating of a viscous liquid flowing under stress, and the reversible mechanocaloric effect in the linear approximation. Formally, this set of equations coincide with the synergetic Lorentz system [20, 21], where the shear stress serves as the order parameter, the conjugate field is reduced to the shear strain, and the temperature is a control parameter. As is known, this system is used for describing thermodynamic and kinetic phase transformations.

In [13], melting of an ultrathin lubricant film between atomically smooth rubbing mica surfaces is viewed as a result of shear stresses spontaneously arising upon heating of rubbing surfaces above critical temperature $T_{\mathrm{c} 0}=1+g^{-1}$. The initial reason for self-organization is positive feedback of $T$ and $\sigma$ with $\varepsilon$ (see (3)) due to the temperature dependence of the shear viscosity, which causes its divergence. However, negative feedback of $\sigma$ and $\varepsilon$ with $T$ (see (4)) is also of importance, since it makes the system stable.

In terms of such an approach, a lubricant is seen as a high-viscosity liquid behaving like an amorphous solid: it has a very high effective viscosity and still can be characterized by a yield stress $[2,19]$. In the solidlike state, shear stresses $\sigma=0$, since Eq. (2), describing the elastic properties in the steady state $(\dot{\sigma}=0)$, is omitted from consideration. Equation (3), containing viscous stresses, reduces to the Debye equation, which describes fast relaxation of the shear strain within the microscopic time $\tau_{\varepsilon} \approx a / c \sim 10^{-12} \mathrm{~s}$, where $a \sim 1 \mathrm{~nm}$ is the lattice constant or molecular spacing and $c \sim$ $10^{3} \mathrm{~m} / \mathrm{s}$ is the speed of sound. In this case, Eq. (4) turns into the simplest expression for temperature relaxation, which is free of the terms corresponding to dissipative heating and the mechanocaloric effect for a viscous liquid.

If stresses $\sigma$ are nonzero, Eqs. (2)-(4) describe all the above properties for the liquid-like state of the lubricant. Moreover, if the shear strain is absent, the rms thermal displacement of atoms (molecules) is given by $\left\langle u^{2}\right\rangle=T / G a$ [6]. The rms displacement due to shear is found from the expression $\left\langle u^{2}\right\rangle=\sigma^{2} a^{2} / G^{2}$. The total rms displacement is a sum of these two displacements provided that temperature fluctuations and stresses are mutually independent. This means that melting of a lubricant is caused by both heating and stresses generated by rubbing surfaces. This supposition is consistent with the concept of dynamic shear melting, according to which the solid-like state is unstable in the absence of temperature fluctuations. Thus, strain (stress) fluctuations and temperature fluctuations should be considered separately. We will assume that, as the temperature grows, the film becomes progressively closer to the liquid state and the friction force decreases as a result of a decrease in the molecular jump activation energy. In addition, the friction force 


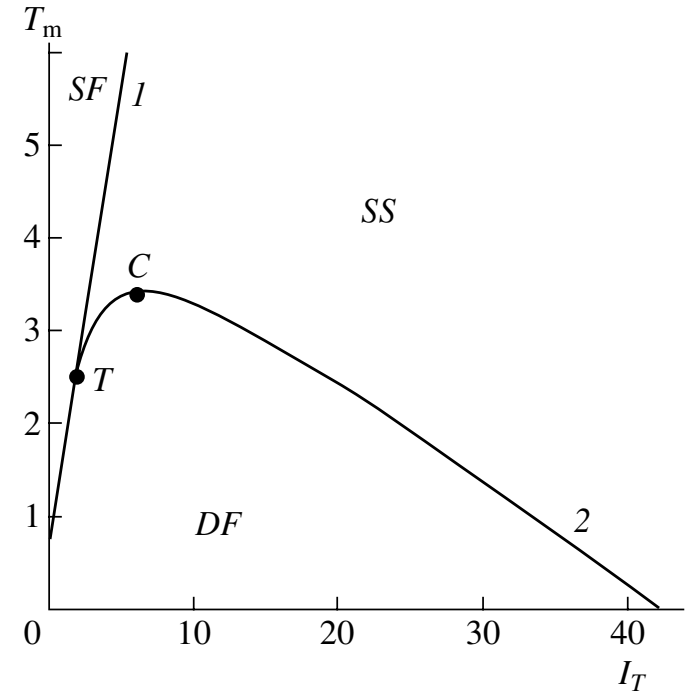

Fig. 1. Phase diagram for $g=0.5$ and $I_{\varepsilon}=1.2$. Curves 1 and 2 are the boundaries between the domains of stable SF, DF, and SS ( $T$ and $C$ are the tricritical and critical points, respectively).

decreases with increasing the velocity of relative motion of contacting surfaces, $V=l \partial \varepsilon / \partial t$, since an increase in the relative velocity causes a rise in shear stresses according to the Maxwell-type relationship $\partial \sigma / \partial t=-\sigma / \tau_{\sigma}+G \partial \varepsilon / \partial t$ between stress and strain.

In macroscopic Lorentz equations (2)-(4), stress $\sigma$, strain $\varepsilon$, and temperature $T$ are averaged over a physically small volume. Fluctuations, which arise over distances on the order of the heat conduction length, will be taken into account by introducing stochastic terms in the form of additive noise intensities $I_{\sigma}^{1 / 2} \xi, I_{\varepsilon}^{1 / 2} \xi$, and $I_{T}^{1 / 2} \xi$ into the right-hand sides of Eqs. (2)-(4) (here, intensities $I_{\sigma}, I_{\varepsilon}$, and $I_{T}$ are given in terms of $\sigma_{s}^{2}, \varepsilon_{s}^{2} \tau_{\varepsilon}^{-2}$, and $\left(T_{\mathrm{c}} \kappa / l\right)^{2}$, respectively, and $\xi(t)$ is a $\delta$-correlated stochastic function $[14,22])$. According to experimental data for organic lubricants [2], the stress relaxation time under normal pressure is $\tau_{\sigma} \sim 10^{-10} \mathrm{~s}$ and increases by several orders of magnitude under a high pressure. Since the ultrathin lubricant film is less than four molecular layers thick in our case, the temperature relaxes to value $T_{\mathrm{m}}$ for time $\tau_{\mathrm{h}}$ that satisfies the inequality $\tau_{\mathrm{h}} \ll \tau_{\sigma}$. Then, in the adiabatic approximation $\tau_{\sigma} \gg$ $\tau_{\varepsilon}$ and $\tau_{\mathrm{h}}[20,21]$, Eqs. (2)-(4) take the form of the Langevin equation [14]

$$
\tau_{\sigma} \dot{\sigma}=f(\sigma)+\sqrt{I(\sigma)} \xi(t), \quad f \equiv-\frac{\partial V}{\partial \sigma},
$$

where force $f$ is specified by the synergetic potential

$$
V=\frac{1}{2}(1-g) \sigma^{2}+g\left(1-\frac{T_{\mathrm{m}}}{2}\right) \ln \left(1+\sigma^{2}\right)
$$

and the effective noise intensity is given by expression

$$
I(\sigma) \equiv I_{\sigma}+\left(I_{\varepsilon}+I_{T} \sigma^{2}\right) g^{2} d^{2}(\sigma),
$$

which follows from the additivity of dispersions of Gaussian random quantities [22]. The stationary distribution of solutions to Eq. (5),

$$
P(\sigma)=Z^{-1} \exp \{-U(\sigma)\},
$$

depends on normalizing factor $Z$ and effective potential

$$
U(\sigma)=\ln I(\sigma)-\int_{0}^{\sigma} \frac{f\left(\sigma^{\prime}\right)}{I\left(\sigma^{\prime}\right)} d \sigma^{\prime}, \quad f \equiv-\frac{\partial V}{\partial \sigma},
$$

where $V$ is synergetic potential (6) and $I(\sigma)$ is noise intensity (7) [23].

The equation defining the positions of the maxima of distribution function $P(\sigma)$ has the form

$$
\begin{gathered}
(1-g) x^{3}+g\left(2-T_{\mathrm{m}}\right) x^{2}-2 g^{2} I_{T} x+4 g^{2}\left(I_{T}-I_{\varepsilon}\right)=0, \\
x \equiv 1+\sigma^{2} .
\end{gathered}
$$

Thus, distribution $P(\sigma)$ does not depend on noise intensity $I_{\sigma}$ and stresses $\sigma$ : it is specified by temperature $T_{\mathrm{m}}$ of rubbing mica surfaces; noise intensities $I_{\varepsilon}$ and $I_{T}$ for strain $\varepsilon$ and lubricant film temperature $T$, respectively; and parameter $g$.

With intensity $I_{\varepsilon}$ fixed, the phase diagram has the form shown in Fig. 1, where lines 1 and 2 are those lines where the system loses stability. Above line 1 defined by the equality

$$
T^{c}=1+g^{-1}+2 g\left(I_{T}-2 I_{\varepsilon}\right),
$$

the condition $\sigma \neq 0$ is the most plausible and so the lubricant is in the liquid-like state, which provides stable sliding friction (SF) and, accordingly, sliding of the surfaces. Below curve 2, which touches straight line 1 in tricritical point $T$ with the coordinates

$$
\begin{gathered}
T_{\mathrm{m}}^{c}=\frac{2}{3}\left(1+2 g^{-1}-2 g I_{\varepsilon}\right), \\
I_{T}^{c}=\frac{1}{6 g}\left(g^{-1}-1+8 g I_{\varepsilon}\right),
\end{gathered}
$$

function $P(\sigma)$ has a maximum only at $\sigma=0$ and we are dealing with dry friction (DF), which is typical of a solid-like lubricant film. Between these lines, where $P(\sigma)$ has maxima at both zero and nonzero stresses, there lies the domain of stick-slip (SS) friction, where the SF-DF and DF-SF transitions periodically occur. Such transitions characterize intermittent melting of the lubricant, when it represents a mixture of liquid- and solid-like states. According to (12), such a scenario is possible even when temperature $T_{\mathrm{m}}$ of rubbing surfaces is zero provided that the amount of strain fluctuations exceeds the critical value $I_{\varepsilon}=\left(1+2 g^{-1}\right) / 2 g$. Under these conditions, the system behaves as under the SOC conditions [15]. 


\section{SELF-SIMILARITY CONDITIONS}

To further investigate the system, it is necessary to find probability distribution (8), which is specified by effective potential (9). Temperature fluctuations $\left(I_{T} \gg I_{\varepsilon}\right.$ and $I_{\sigma}$ ) lead us to the expression

$$
\begin{gathered}
P(\sigma) \approx I_{T}^{-1}[g \sigma d(\sigma)]^{-2} \\
\times \exp \left\{I_{T}^{-1} g^{-2} \int f(\sigma)[\sigma d(\sigma)]^{-2} d \sigma\right\}, \\
f(\sigma)=-\sigma(1-g)+g\left(T_{\mathrm{m}}-2\right) \sigma d(\sigma), \\
d(\sigma) \equiv\left(1+\sigma^{2}\right)^{-1} .
\end{gathered}
$$

Since the integral in (13) tends to a constant in the limit $\sigma \longrightarrow 0$ and $d(\sigma) \longrightarrow 1$, distribution (13) has a power-type asymptotics, $P(\sigma) \sim \sigma^{-2}$. Thus, self-similar conditions without a characteristic stress scale are established that are specified by the homogeneous function

$$
P(y)=y^{-2 a} \mathscr{P}(\sigma), \quad y=\sigma \sigma_{s}
$$

with integer exponent $2 a=2$ [24].

However, this exponent may be fractional in the general case; specifically, the SOC conditions are characterized by $2 a=1.5$. To avoid loss in generality, we replace order parameter $\sigma$ by $\sigma^{a}(0 \leq a \leq 1)$ in all the terms of Eqs. (2)-(4). Then, with regard to stochastic additions, the basic equations in dimensionless variables take the form

$$
\begin{gathered}
\tau_{\sigma} \dot{\sigma}=-\sigma^{a}+g \varepsilon+\sqrt{I_{\sigma}} \xi(t), \\
\tau_{\varepsilon} \dot{\varepsilon}=-\varepsilon+(T-1) \sigma^{a}+\sqrt{I_{\varepsilon}} \xi(t), \\
\tau_{\mathrm{h}} \dot{T}=\left(T_{\mathrm{m}}-T\right)-\sigma^{a} \varepsilon+\sigma^{2 a}+\sqrt{I_{T}} \xi(t) .
\end{gathered}
$$

Physically, such a replacement of the exponent means that self-similarity is achieved under the assumption that stress relaxation is nonlinear and both positive and negative feedbacks are of fractional character. The adiabaticity conditions $\left(\tau_{\varepsilon}, \tau_{\mathrm{h}} \ll \tau_{\sigma}\right)$ immediately lead us to the Langevin equation (cf. (5))

$$
\tau_{\sigma} \dot{\sigma}=f_{a}(\sigma)+\sqrt{I_{a}(\sigma)} \xi(t),
$$

where force $f_{a}(\sigma)$ and noise intensity $I_{a}(\sigma)$ are given by

$$
\begin{gathered}
f_{a}(\sigma) \equiv-\sigma^{a}+g \sigma^{a}\left[1-\left(2-T_{\mathrm{m}}\right) d_{a}(\sigma)\right], \\
I_{a}(\sigma) \equiv I_{\sigma}+g^{2}\left(I_{\varepsilon}+I_{T} \sigma^{2 a}\right) d_{a}^{2}(\sigma), \\
d_{a}(\sigma) \equiv\left(1+\sigma^{2 a}\right)^{-1} .
\end{gathered}
$$

The corresponding distribution (cf. (8)),

$$
P_{a}(\sigma)=\frac{Z^{-1}}{I_{a}(\sigma)} \exp \left(-V_{a}(\sigma)\right)
$$

is specified by partition function $Z$ with the effective potential

$$
V_{a}(\sigma) \equiv-\int_{0}^{\sigma} \frac{f_{a}\left(\sigma^{\prime}\right)}{I_{a}\left(\sigma^{\prime}\right)} d \sigma^{\prime} .
$$

The extreme points of this distribution are found from the equation

$$
\frac{T_{\mathrm{m}}-2}{1+\sigma^{2 a}}+\frac{2 a g \sigma^{a-1}}{\left(1+\sigma^{2 a}\right)^{3}}\left[2 I_{\varepsilon}-I_{T}\left(1-\sigma^{2 a}\right)\right]=\frac{1-g}{g},
$$

according to which the boundary of the SF domain,

$$
I_{T}=2 I_{\varepsilon},
$$

meets the condition $\sigma=0$. Expressions (22) and (23) are extensions of equalities (10) and (11). It follows from the above expressions that the results obtained at $a=1$ differ little from those obtained in the general case $0 \leq a \leq 1$. In particular, the effect of the random stress distribution is, as before, insignificant, while strain and temperature fluctuations have a crucial effect. The dependence of stationary shear stresses $\sigma_{0}$ on temperature $T_{\mathrm{m}}$ changes most drastically. In the stationary determinate case, set (15)-(17) has the solution

$$
\sigma_{0}=\left[g(1-g)^{-1}\left(T_{\mathrm{m}}-2\right)-1\right]^{1 / 2 a}, \quad g<1,
$$

which generalizes the standard root dependence in the case $a=1$ (Fig. 2). As strain noise $I_{\varepsilon}$ increases, $\sigma_{0}$ monotonically grows. A rise in $I_{T}$ produces a barrier near $\sigma_{0}=0$. In addition, the dependence $\sigma_{0}\left(T_{\mathrm{m}}\right)$ becomes nonmonotonic when $I_{T}$ lies above straight line (23) (Fig. 3). The dashed lines in Fig. 3 cover unstable values of stresses $\left(\sigma^{m}\right)$; the continuous lines and portions of lines, stable values $\left(\sigma_{0}\right)$. It follows from Fig. 3 that $\sigma^{m}$ may take a zero value only at $a=1$ or $I_{T}=2 I_{\varepsilon}$; otherwise, the curves $\sigma^{m}\left(T_{\mathrm{m}}\right)$ asymptotically tend to

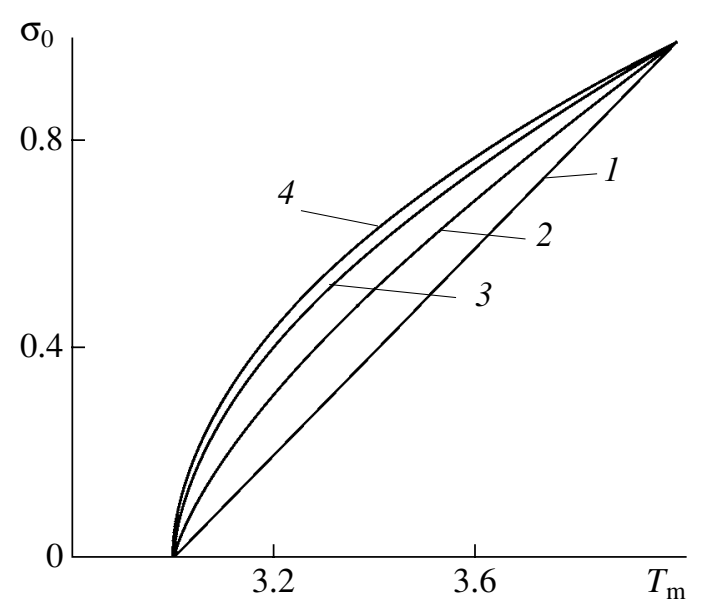

Fig. 2. Shear stresses $\sigma_{0}$ vs. temperature $T_{\mathrm{m}}$ for $g=0.5$ and $a=$ (1) 0.5, (2) 0.7, (3) 0.9, and (4) 1.0. 

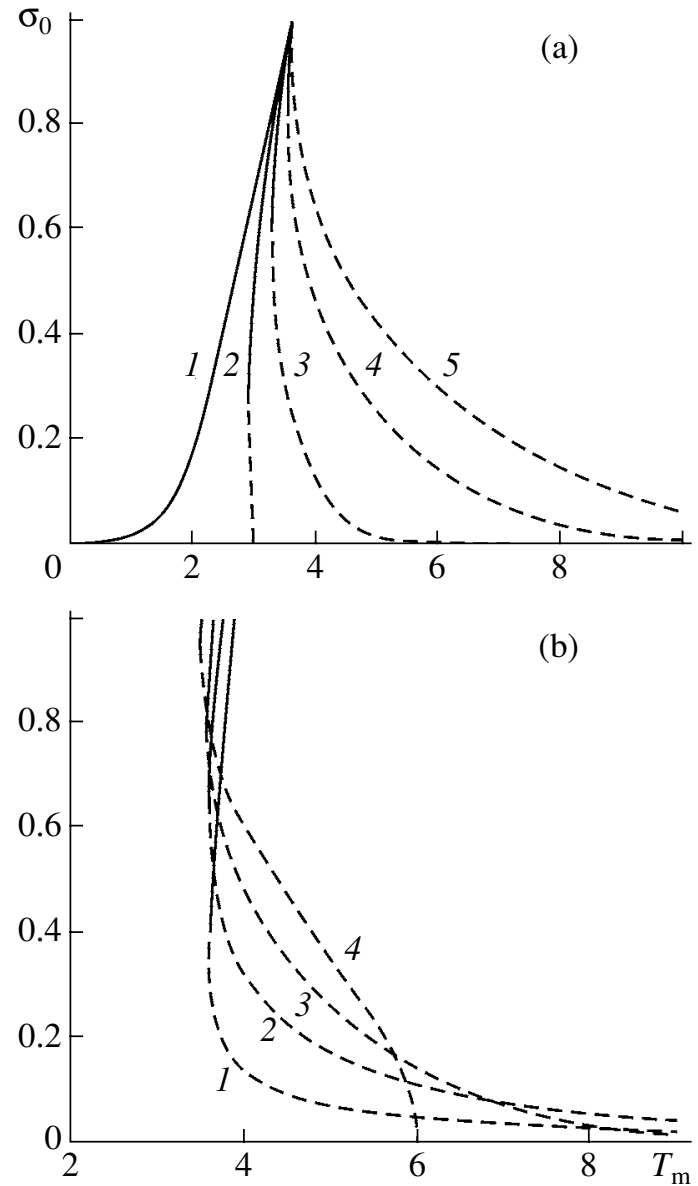

Fig. 3. Shear stresses $\sigma_{0}$ vs. temperature $T_{\mathrm{m}}$ for $g=0.5$. (a) $a=0.75 ; I_{\varepsilon}=1$; and $I_{T}=$ (1) 1 , (2) 2, (3) 3, (4) 5 , and (5) 7. (b) $I_{\varepsilon}=1 ; I_{T}=5$; and $a=(1) 0.25$, (2) 0.50, (3) 0.75, and (4) 1.00 .

zero. This means that, if $I_{T}>2 I_{\varepsilon}$ and $a \neq 1$, there always exists effective potential barrier (21) near the point $\sigma_{0}=$ 0 . In other words, either the lubricant experiences the first-order liquid-solid transition or the DF conditions set in. At $I_{T}<2 I_{\varepsilon}$ and $a \neq 1$, SF occurs, which corresponds to a minimum of the potential with $\sigma_{0} \neq 0$, since the barrier reaches a maximum in the physically meaningless domain $\sigma^{m}<0$.

The phase diagram illustrating the state of the system at different noise intensities $I_{\varepsilon}$ and $I_{T}$ (Fig. 4) is similar to that corresponding to the case $a=1$. As $a$ grows, the two-phase SS domain, which is bounded by straight line (23) and a bell-shaped curve, expands. When $a=1$, the SF regime at small $I_{\varepsilon}$ does not occur, unlike in the case $a \neq 1$. In practice, the noise intensity is, as a rule, low, and so friction is expected to reduce in systems with fractional exponent $a$.

Figure 5 shows probability distribution (20) corresponding to the points marked in Fig. 4. The positions of maxima in this distribution are specified by a set of parameters $I_{\varepsilon}, I_{T}, I_{\sigma}, a, g$, and $T_{\mathrm{m}}$. For point $l$ in the two-

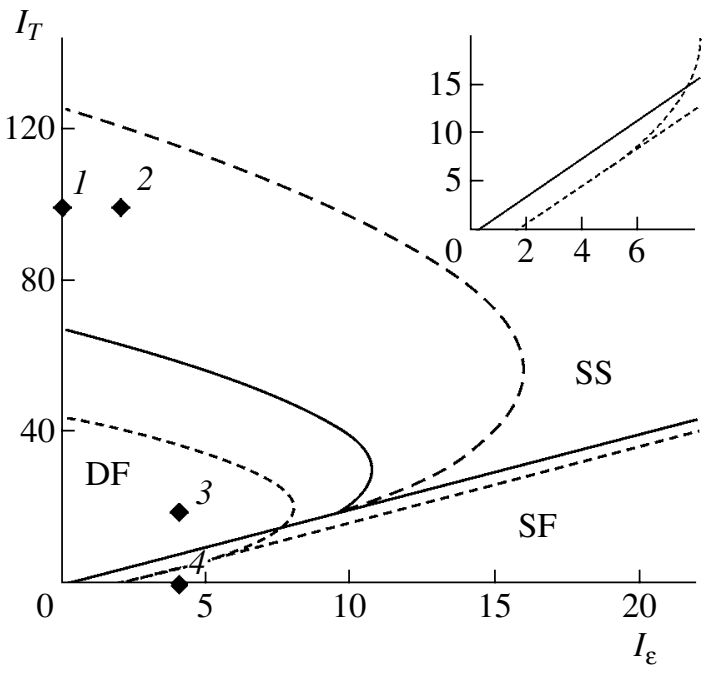

Fig. 4. Phase diagram of the system for $T_{\mathrm{m}}=0 ; g=0.5$; $I_{T}$ and $I_{\varepsilon} \neq 0$; and $a=0.50$ (dashed curve), 0.75 (continuous curve), and 1.00 (dotted curve).

phase SS domain, the distribution obeys a power law (typical of the SOC conditions) with external action $T_{\mathrm{m}}=0$. Such conditions correspond to $\sigma \ll 1, I_{\sigma}$, and $I_{\varepsilon}$ $\ll I_{T}$. With such values of these parameters, Eq. (20) reduces to canonical form (14), in which function $\mathscr{P}(\sigma)$ is given by

$$
\begin{gathered}
\mathscr{P}(\sigma)=Z^{-1} g^{-2} I_{T}^{-1} d_{a}^{-2}(\sigma) \\
\times \exp \left\{-I_{T}^{-1} g^{-2} \int_{0}^{\sigma} \frac{1-g\left[1-2 d_{a}\left(\sigma^{\prime}\right)\right]}{d_{a}^{2}\left(\sigma^{\prime}\right)\left(\sigma^{\prime}\right)^{a}} d \sigma^{\prime}\right\} .
\end{gathered}
$$

At point 2, distribution $P_{a}(\sigma)$ has maxima at both zero and nonzero stresses. Hence, point 2 lies in the SS domain. Point 3 belongs to the DF domain, where $P_{a}(\sigma)$ has a single maximum at $\sigma_{0}=0$. Finally, point 4 lies in the domain where the probability distribution has a single maximum at $\sigma_{0} \neq 0(\mathrm{SF})$.

\section{FRACTAL AND NONADDITIVE NATURE OF FRICTION}

A feature of distribution (25) is that it is expressed through integral $\mathscr{T}_{-\sigma}^{1-a}$ of fractional power $1-a$ (see the Appendix),

$$
\begin{gathered}
\mathscr{P}(\sigma)=Z^{-1} g^{-2} I_{T}^{-1} d_{a}^{-2}(\sigma) \\
\times \exp \left\{-\frac{\Gamma(1-a)}{I_{T} g^{2}} \mathscr{T}_{-\sigma}^{1-a}\left\{d_{a}^{-2}(\sigma)\left[1-g\left(1-2 d_{a}(\sigma)\right)\right]\right\}\right\},
\end{gathered}
$$

where $\Gamma(x)$ is the gamma function. 
At the same time, it is known [18] that an expression of this type is a solution to the fractional Fokker-Planck equation

$$
\begin{aligned}
& \mathscr{D}_{t}^{\omega} \mathscr{P}(\sigma, t)=\mathscr{D}_{-\sigma}^{\bar{\omega}}\left\{\sigma \mathscr{P}(\sigma, t)+\frac{I_{T} g^{2}}{\Gamma(\bar{\omega})} \mathscr{D}_{-\sigma}^{\bar{\omega}}\right. \\
& \left.\quad \times\left\{d_{a}^{2}(\sigma)\left[1-g\left(1-2 d_{a}(\sigma)\right)\right]^{-1} \mathscr{P}(\sigma, t)\right\}\right\},
\end{aligned}
$$

where fractional derivative $\mathscr{D}_{x}^{\bar{\omega}}$ (see A.2) implies the operation inverse to fraction integration (A.1).

Let us multiply equality (27) by $\sigma^{2 \bar{\omega}}$ and average the result over $\sigma$. Then, for the average

$$
\begin{gathered}
|\sigma| \equiv\left\langle\sigma^{\alpha}\right\rangle^{\frac{1}{\alpha}}, \\
\left\langle\sigma^{\alpha}\right\rangle \equiv \int_{-\infty}^{+\infty} \sigma^{\alpha} \mathscr{P}(\sigma, t) d \sigma, \quad \alpha>0
\end{gathered}
$$

with fraction order $\alpha \equiv 2 \bar{\omega}$, we obtain

$$
|\sigma|^{z} \sim t, \quad z=\frac{2 \bar{\omega}}{\omega},
$$

where $z$ is the dynamic exponent.

Here, we take into account the diffusion contribution alone, which dominates in the long time limit. Combining equalities (26), (29), and (A.1) yields 1 $a=\bar{\omega}=z \omega / 2$, or

$$
a=1-\frac{z \omega}{2} .
$$

In the mean field approximation, exponent $a$ in (14) is $a=3 / 4$. Then, from expression (30), we have

$$
\omega z=\frac{1}{2}
$$

At $\omega=1$, which corresponds to dynamic exponent $z=1 / 2$, the system evolves without traps in the phase space. According to (29), this value of $z$ is smaller than $z=1$, which is characteristic of ballistic behavior. On the other hand, fractional Fokker-Planck equation (27) leads to the diffusion conditions corresponding to $z=2$ only when the order of the time derivative is $\omega=1 / 4$.

Thus, in the mean field approximation $(a=3 / 4, \bar{\omega}=$ $1 / 4$ ), stick-slip friction with effective traps in the phase space is established when the order of the time derivative lies in the range $1 / 4<\omega<1 / 2$ and dynamic exponent $z$ falls into the range $1<z<2$. Essentially, such a situation is peculiar to the subdiffusion process, when the displacement of a walking particle is continuous in space but occurs discretely (at particular time instants); hence, the order of the corresponding derivative is fractional, $\omega<1$. Unlike this situation, the walk of a particle

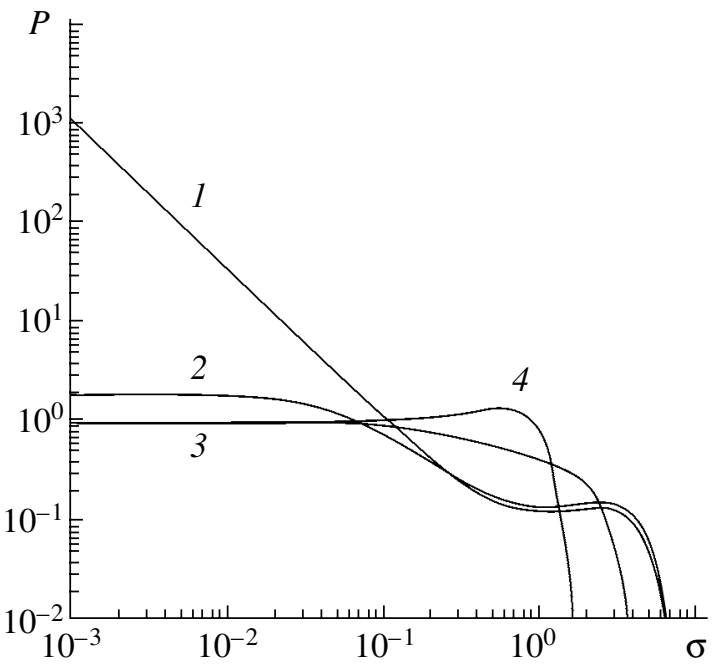

Fig. 5. Distribution function (20) for $a=0.75, g=0.5$, $T_{\mathrm{m}}=0, I_{\sigma}=0$, and conditions shown by points in Fig. 4: (1) $I_{\varepsilon}=0$ and $I_{T}=100$ (SOC), (2) $I_{\varepsilon}=2$ and $I_{T}=100$ (SS), (3) $I_{\varepsilon}=4$ and $I_{T}=20(\mathrm{DF})$, and (4) $I_{\varepsilon}=4$ and $I_{T}=0(\mathrm{SF})$.

at Levi flights (arbitrary, including infinite, displacements) proceeds continuously in time but discretely in space [25]. In accordance with Fokker-Planck equation (27), the Levi process is characterized by order $\omega=1$ and order $\bar{\omega} \leq 1$, the latter being the order of the fractional derivative with respect to the particle coordinate. To avoid confusion, it should be noted that we consider hopping in the phase, rather than in the real geometrical, space.

Following [17], let us analyze the system in terms of nonadditive thermodynamics. It will be assumed that strain is converted to the system's complexity, which is a measure of disorder and, by analogy with the Tsallis entropy, is expressed as

$$
S^{(q)} \equiv \frac{1-\sum_{i} p_{i}^{q}}{q-1},
$$

where $q \neq 1$ is the nonadditivity parameter. ${ }^{1}$ Also, the temperature of the lubricant film is replaced by its internal energy given by

$$
\xi^{(q)} \equiv \sum_{i} \xi_{i} Q_{i}
$$

\footnotetext{
${ }^{1}$ In the limit $q \longrightarrow 1$, expression (32) turns into the formula for conventional entropy, $S\left(p_{i}\right)=S^{(1)}\left(p_{i}\right)=-\Sigma_{1} p_{i} \ln p_{i}$. Considering two independent subsystems $A$ and $B$ yields $p_{i j}^{A B}=p_{i}^{A} p_{j}^{B}$ for the probability and $S_{A B}^{(q)}=S_{A}^{(q)}+S_{B}^{(q)}+(1-q) S_{A}^{(q)} S_{B}^{(q)}$ for the entropy. The latter does not possess the additivity property $S_{A B}=$ $S_{A}+S_{B}$, unlike the entropy in the conventional sense.
} 
where distributions $Q_{i} \equiv p_{i}^{q} / \sum_{j} p_{j}^{q}$ and $\left\{\xi_{i}\right\}$ are the eigenvalues of the corresponding Hamiltonian that are obtained in view of boundary conditions [26].

Such a parametrization allows us to relate exponent $z$, order $\bar{\omega}$, and order $\omega$ with parameter $q$ specifying expressions (32) and (33) [17]. The evolution of a nonadditive system is represented by the nonlinear FokkerPlanck equation

$$
\mathscr{D}_{t}^{\omega} P(\sigma, t)=\mathscr{D}_{-\sigma}^{2} P^{q}(\sigma, t)
$$

where order $\omega$ and exponent $q$ are fractional, $\mathscr{D}_{t}^{\omega}$ is the fractional time derivative, and the units of measure are taken so as to exclude the effective diffusion coefficient [27].

For the self-similar normalized function

$$
\begin{gathered}
P(\sigma, t)=\sigma_{\mathrm{c}}^{-1} \mathscr{P}(x) ; \\
\sigma_{\mathrm{c}} \equiv \sigma_{\mathrm{c}}(t), \quad x \equiv \sigma / \sigma_{\mathrm{c}},
\end{gathered}
$$

where $\sigma_{\mathrm{c}}$ is the critical value [28], we have

$$
\sigma_{\mathrm{c}}^{1+q} \sim t^{\omega}, \quad \mathscr{P}^{q-1} \sim x^{2} .
$$

At the same time, the linear fractional FokkerPlanck equation (cf. (27))

$$
\mathscr{D}_{t}^{\omega} P(\sigma, t)=\mathscr{D}_{-\sigma}^{2 \bar{\omega}} P(\sigma, t)
$$

yields [29]

$$
\sigma_{\mathrm{c}}^{2 \bar{\omega}} \sim t^{\omega}, \quad \mathscr{P} \sim\left\{\begin{array}{l}
x^{2 \bar{\omega}-1}, \quad x \longrightarrow 0 \\
x^{-(1+2 \bar{\omega})}, \quad x \longrightarrow \infty .
\end{array}\right.
$$

Comparing the first expressions in (36) and (38) gives the relationship

$$
1+q=2 \bar{\omega} .
$$

Since the mean value of $|\sigma|$ in (28) is on the order of $\sigma_{\mathrm{c}}$ for self-similar systems, we find from (29), (36), and (38) that

$$
1+q=z \omega .
$$

From the above consideration, it follows that product $z \omega \leq 1$ is typically less than unity (specifically, in the mean field approximation, we have (31)); so, condition (40) holds only if $-1<q<0$. Thus, the given thermodynamic system is superadditive $(q<1)$ : the total entropy exceeds the sum of partial entropies.

Fractional Lorentz system (15)-(17) can be assigned a fractal phase space. To complete the analysis, let us find a relationship between its fractal dimension $D$ and the exponents and orders of derivative introduced above. To do this, we take advantage of the standard scaling relationships [28]

$$
a=\frac{1}{2}\left(1+\frac{z}{D}\right), \quad a=1-D^{-1} .
$$

Comparing the last of them with (30), we find that

$$
\omega z=\frac{2}{D} \text {. }
$$

To calculate $D$, one should take into account that each of the stochastic degrees of freedom $\left(\sigma, S^{(q)}\right.$, and $\left.\xi^{(q)}\right)$, the number of which is $n=3$, is assigned a conjugate momentum; therefore, a smooth phase space must have dimension $D=2 n$. Such a space arises in the absence of feedback (the simplest case), when its related exponent $a=0$ and noise is additive. As exponent $a$ (which specifies the effective force and noise intensity in relationships (19)) grows $(a>0)$, so does the amount of feedback and the fluctuations (noise) become multiplicative. Accordingly, the phase space becomes fractal [23] and its dimension decreases by $(1-a)$ times. Eventually, the dimension of the space where a self-organizing system evolves becomes equal to

$$
D=2 n(1-a),
$$

where $n=3$ for the Lorentz system.

In the general case, using equalities (30), (42), and (43), one can find the number of self-consistent stochastic equations to describe SOC at different feedback exponents,

$$
n=\frac{1}{2(1-a)^{2}} \text {. }
$$

In the range of interest $(a \leq 1), n$ indefinitely grows, starting from the minimal physical value $n_{\mathrm{c}}=1$, which corresponds to $a=1-1 / \sqrt{2}$. Such a one-parametric case was considered in [30]. With a further increase in $a$, the number of degrees of freedom needed to describe SOC should be augmented. In particular, the case $a=$ $1 / 2$ corresponds to a two-parametric representation of a self-organizing system [28, 31]. For the Lorentz system $(n=3[21,32])$ to be considered, deeper feedback, $a=$ $1-1 / \sqrt{6}$, is necessary.

Combining equalities (40), (42), and (43), we come to a final expression for the nonadditivity parameter,

$$
1+q=\frac{1}{n(1-a)} .
$$

Substituting expression (44) for number $n$ of equations needed to represent the SOC conditions into (45) yields $q=1-2 a$. Hence, as the amount of feedback reduces ( $a$ declines), parameter $q$ grows. This parameter tends to a maximal value $(q \longrightarrow 1)$ in systems without feedback $(a \longrightarrow 0)$. Thus, provided that scaling relationships (41) from the mean field theory are valid, (i) one can reproduce the results obtained with various approaches [33], using expression (44), and (ii) the thermodynamic system under study is superadditive.

The above consideration has demonstrated that stick-slip friction can be described in terms of the con- 
cept of SOC. Under the SOC conditions, the film melts at the zero temperature of rubbing surfaces. The basic feature of these conditions is that the system evolves in a self-similar manner and, accordingly, its distribution function has power-type asymptotics. It has also been shown that this fact is embodied in the Lorentz system parametrized by shear stresses, strain, and lubricant film temperature. With the self-similarity conditions taken into account, stress relaxation and feedback in the Lorentz system acquire a fractional character. The associated phase diagram separating out the SF, SS, and DF domains qualitatively coincides with that constructed for the case when this relaxation term and feedbacks are free of a fractional exponent. It should be noted that, when this exponent is other than unity, friction can be reduced if the noise intensity is low. For a system parametrized by stresses, complexity, and internal energy, the fractional Lorentz model allows one to relate the exponent in the stress distribution (multiplicative noise), fractal dimension of the phase space, number of equations needed to represent the system under the SOC conditions, and Tsallis nonadditivity parameter. Finally, it has been demonstrated that stickslip friction is due to effective traps present in the phase space and can be identified with subdiffusion, which one may speak of when the order of the time derivative in the fractional Fokker-Planck equation is less than unity $(\omega<1)$.

\section{ACKNOWLEDGMENTS}

This work was supported by a grant of the cabinet of Ukraine.

APPENDIX

The integral of fractional order $(\bar{\omega}>0)$ is defined as [34]

$$
\mathscr{T}_{x}^{\overline{\bar{\omega}}} f(x) \equiv \frac{1}{\Gamma(\bar{\omega})} \int_{0}^{x} \frac{f\left(x^{\prime}\right)}{\left(x-x^{\prime}\right)^{1-\bar{\omega}}} d x^{\prime},
$$

where $f(x)$ is an arbitrary function and $\Gamma(x)$ is the gamma function.

The operation inverse to such integration, $\mathscr{D}_{x}^{\bar{\omega}} \equiv$ $\mathscr{T}_{x}^{-\bar{\omega}}$, is called fractional differentiation of order $\bar{\omega}>0$,

$$
\mathscr{D}_{x}^{\bar{\omega}} f(x) \equiv \frac{1}{\Gamma(-\bar{\omega})} \int_{0}^{x} \frac{f\left(x^{\prime}\right)}{\left(x-x^{\prime}\right)^{1+\bar{\omega}}} d x^{\prime} .
$$

In the range $0<\bar{\omega}<1$, it is convenient to use the expression

$$
\mathscr{D}_{x}^{\overline{\bar{\omega}}} f(x) \equiv \frac{\bar{\omega}}{\Gamma(1-\bar{\omega})} \int_{0}^{x} \frac{f(x)-f\left(x^{\prime}\right)}{\left(x-x^{\prime}\right)^{1+\bar{\omega}}} d x^{\prime},
$$

which takes into account that $x \Gamma(x)=\Gamma(x+1)$ for $x \equiv-\bar{\omega}$.

\section{REFERENCES}

1. B. N. J. Persson, Sliding Friction: Physical Principles and Applications (Springer, Berlin, 1998).

2. H. Yoshizawa, Y.-L. Chen, and J. Israelachvili, J. Phys. Chem. 97, 4128 (1993); H. Yoshizawa and J. Israelachvili, J. Phys. Chem. 97, 11300 (1993).

3. E. D. Smith, M. O. Robbins, and M. Cieplak, Phys. Rev. B 54, 8252 (1996).

4. J. Krim, D. H. Solina, and R. Chiarello, Phys. Rev. Lett. 66, 181 (1991).

5. J. M. Carlson and A. A. Batista, Phys. Rev. E 53, 4153 (1996).

6. I. S. Aranson, L. S. Tsimring, and V. M. Vinokur, Phys. Rev. B 65, 125402 (2002).

7. P. A. Thompson and M. O. Robbins, Phys. Rev. A 41, 6830 (1990).

8. F. Family, M. O. Braiman, and H. G. E. Hentschel, Friction, Arching, Contact Dynamics, Ed. by D. E. Wolf and P. Grassberger (World Sci., Singapore, 1996), pp. 33-41.

9. Y. Braiman, H. G. E. Hentschel, F. Family, et al., Phys. Rev. E 59, R4737 (1999).

10. O. M. Braun and Yu. S. Kivshar, Phys. Rev. B 43, 1060 (1991).

11. J. B. Sokoloff, Phys. Rev. B 51, 15573 (1995).

12. T. Kawaguchi and H. Matsukawa, Phys. Rev. B 56, 4261 (1997).

13. A. V. Khomenko and O. V. Yushchenko, Phys. Rev. E 68, 036110 (2003).

14. A. V. Khomenko, Phys. Lett. A 329, 140 (2004).

15. P. Bak, How Nature Works: The Science of Self-Organized Criticality (Springer, New York, 1996).

16. A. I. Olemskoi, Physica A 310, 223 (2002).

17. C. Tsallis, in Lecture Notes in Physics, Ed. by S. Abe and Y. Okamoto (Springer, Heidelberg, 2001).

18. G. M. Zaslavsky, Phys. Rep. 371, 461 (2002).

19. Rheology: Theory and Applications, Ed. by F. R. Eirich (Academic, New York, 1956; Inostrannaya Literatura, Moscow, 1962).

20. H. Haken, Synergetics: An Introduction (Springer, Berlin, 1977; Mir, Moscow, 1980).

21. A. I. Olemskoŭ and A. V. Khomenko, Zh. Éksp. Teor. Fiz. 110, 2144 (1996) [JETP 83, 1180 (1996)].

22. H. Risken, The Fokker-Planck Equation: Methods of Solution and Applications, 2nd ed. (Springer, Berlin, 1989).

23. A. I. Olemskoi, Usp. Fiz. Nauk 168, 287 (1998) [Phys. Usp. 41, 269 (1998)].

24. D. J. Amit, Field Theory, the Renormalization Group and Critical Phenomena (McGraw Hill, New York, 1978).

25. R. Metzler and J. Klafter, Phys. Rep. 339, 1 (2000). 
26. A. I. Olemskoi, A. V. Khomenko, and D. A. Olemskoi, Physica A 332, 185 (2004).

27. A. I. Olemskoi and D. O. Kharchenko, Physica A 293, 178 (2001).

28. A. Vespignani and S. Zapperi, Phys. Rev. Lett. 78, 4793 (1997); Phys. Rev. E 57, 6345 (1998).

29. A. I. Olemskoi and D. O. Kharchenko, J. Phys. Stud. 6, 253 (2002).

30. T. Hwa and M. Kardar, Phys. Rev. A 45, 7002 (1992).

31. A. Mehta and G. C. Barker, Rep. Prog. Phys. 57, 383 (1994).
32. A. I. Olemskoi and A. V. Khomenko, Phys. Rev. E 63, 036116 (2001).

33. A. Chessa, E. Marinari, A. Vespignani, et al., Phys. Rev. E 57, R6241 (1998).

34. S. G. Samko, A. A. Kilbas, and O. I. Marichev, Fractional Integrals and Derivatives: Theory and Applications (Nauka i Tekhnika, Minsk, 1987; Gordon and Breach, Amsterdam, 1993).

Translated by V. Isaakyan 